\title{
Autophagy and ATP-induced anti-apoptosis in antigen presenting cells (APC) follows the cytokine storm in patients after major trauma
}

\author{
E. Marion Schneider $\cdot$ Sarah Flacke $\cdot$ Fengguang Liu $\cdot$ \\ Myriam R. Lorenz • Patricia Schilling • Max E. Nass • \\ Karl J. Foehr • Markus Huber-Lang • Manfred E. Weiss
}

Received: 2 May 2010 / Accepted: 9 December 2010 / Published online: 13 January 2011

(C) The Author(s) 2011. This article is published with open access at SpringerLink.com

\begin{abstract}
Severe trauma and the systemic inflammatory response syndrome (SIRS) occur as a result of a cytokine storm which is in part due to ATP released from damaged tissue. This pathology also leads to increased numbers of immature antigen presenting cells (APC) sharing properties of dendritic cells (DC) or macrophages $(M \Phi)$. The occurrence of immature APC appears to coincide with the reactivation of herpes virus infections such as Epstein Barr virus (EBV). The aim of this study was the comparative analysis of the ultrastructural and functional characteristics of such immature APC. In addition, we investigated EBV infection/ reactivation and whether immature APC might be targets for natural killers (NK). Significant macroautophagy, mitochondrial degradation and multivesicular body formation together with the identification of herpes virus particles were morphological findings associated with immature APC. Exogenous stressors such as ATP further increased morphological signs of autophagy, including LC3 expression. Functional tests using fluorescent bacteria
\end{abstract}

Electronic supplementary material The online version of this article (doi:10.1007/s12079-010-0113-z) contains supplementary material, which is available to authorized users.

E. M. Schneider $(\bowtie) \cdot$ S. Flacke $\cdot$ F. Liu $\cdot$ M. R. Lorenz $\cdot$

P. Schilling $\cdot$ M. E. Nass $\cdot$ K. J. Foehr $\cdot$ M. E. Weiss

Sektion Experimentelle Anaesthesiologie,

Department of Anesthesiology, University Hospital Ulm,

Steinheovelstr. 9,

89075 Ulm, Germany

e-mail: marion.schneider@uni-ulm.de

M. Huber-Lang

Department Traumatology, Hand-, Plastic- and Reconstructive

Surgery, University Hospital Ulm,

Steinhoevelstr. 9,

89075 Ulm, Germany proved impaired phagolysosome fusion. However, immature APC were susceptible to NK-92-mediated cytolysis. We found evidence for EBV latency state II infection by detecting EBV-specific LMP1 and EBNA2 in immature APC and in whole blood of these patients. In summary, trauma-induced cytokine storms may induce maturation arrest of APC, promote ATP-induced autophagy, support EBV persistence and impair the degradation of phagocytozed bacteria through inefficient phagolysosome fusion. The susceptibility to NK-mediated cytolysis supports the hypothesis that NK function is likely to contribute to immune reconstitution after major trauma by regulating immature APC, and ATP-induced autophagy and survival.

Keywords Autophagy-associated anti-apoptosis · Cultured DC $\cdot$ EBV $\cdot$ Inflammasome $\cdot$ P2RX7 $\cdot$ SIRS

\section{Introduction}

After trauma, damaged cells and tissues release a number of molecules which are members of danger-associated molecular patterns (DAMPs), one of which is ATP (Willart and Lambrecht 2009). ATP may stimulate macrophages as well as dendritic cells via the P2RX7 receptor to release IL-1ß and initiate an inflammatory cascade. IL- $1 \beta$ is able to upregulate TNF- $\alpha$ as well as a number of cellular processes including proliferation, apoptosis and autophagy. Using cell culture techniques we established the enrichment of antigen presenting cell (APC) cultures from patients with severe sepsis and septic shock following a major trauma. For comparison, we isolated a similar cell type from healthy donors' buffy coat cells and studied this cell type by flow cytometry, ultrastructural and functional methods. More- 
over, we addressed the questions whether chronic virus infections play a role and whether these immature APC respond to the danger signal ATP. The susceptibility to NK-mediated cytolysis was investigated by caspase $3 / 7$ measurements of co-cultures with the NK-92 cell line.

According to Hotchkiss and Karl, inflammation is initiated by trauma, leading to a DAMP-induced cytokine storm, which appears to condition for immune nonresponsiveness and culminate in severe sepsis and septic shock (Hotchkiss et al. 2009; Hotchkiss and Karl 2003). Several mechanisms have been proposed which could explain the profound immune suppression associated with multi-organ failure and death. Common denominators of the human disease and experimental animal models have been summarized by Efron and Moldawer (Efron and Moldawer 2003; Efron et al. 2004a,b). The authors also provided evidence that the immune-suppressive dendritic cells constitute major effectors of anergy. A CD11c-positive dendritic cell subpopulation and a high dendritic cell turnover are essential for surviving a septic condition in mice (Scumpia et al. 2005). In contrast, Oberholzer and colleagues favor IL-10-producing immature dendritic cells to be beneficial by attenuating the pro-inflammatory response induced by sepsis (Oberholzer et al. 2005). When comparing systemic inflammatory response syndromes (SIRS) with physical stress syndromes occurring through high endurance exercise, heat shock protein expression appeared to be one parameter of "training against stress" which would facilitate the a non detrimental DAMP signaling response in high endurance exercises but not in trauma associated SIRS (Fehrenbach and Schneider 2006). Importantly, heatshock expression primes NK effectors, which contribute to the above mentioned dendritic cell turnover.

\section{Materials and methods}

\section{Cell culture of patients' APC}

Ficoll-isolated peripheral blood mononuclear cells were depleted from non-adherent cells following $2 \mathrm{~h}$ of plastic adherence. During 3-4 weeks of in vitro culture in RPMI1640 (Invitrogen.com) supplemented with 10\% fetal calf serum (FCS) (selected for a very low endotoxin content, $<2$ endotoxin units (EU)/ml), $25 \mathrm{mM}$ HEPES and antibiotics, a morphologically homogeneous cell population was enriched which expressed markers for APC. The phenotype of these APC is patient specific and can be reproduced by a second cell culture initiated from frozen mononuclear cells of the same isolate. From $5 \mathrm{ml}$ of heparinized blood, $2-3 \times 10^{6}$ mononuclear cells with a proportion of $10-15 \%$ of cells expressing markers for monocytes (CD14+) or dendritic cells (CD11c, CD123/DR) were obtained. The enriched antigen presenting cells per $2-4 \times 10^{5}$ adherent cells did not display a proliferative capacity beyond one or two cell divisions. After $2-4$ weeks of in vitro culture, the cell concentration of $1-1.5 \times 10^{5}$ immature $\mathrm{APC} / \mathrm{ml}$ remained constant. According to propidium iodide exclusion and morphology by Giemsa-stained cytopsin preparations, the viability was $>90 \%$. Overall, cell death was less than 5\% during days $14-40$ of in vitro culture. Immature APC were amoeboid, floating or loosely adherent and could be readily harvested by gentle agitation.

\section{Cell culture of APC derived from healthy donors}

In contrast to patients' adherent cell fractions following Ficoll-isolation, healthy donors' blood samples did not give rise to immature APC cultures. However, when we used buffy coat preparations which were isolated from blood units stored for $24 \mathrm{~h}$ before preparation of erythrocyte concentrate, we were successful in obtaining higher amounts of mononuclear cells $\left(>2 \times 10^{8} \mathrm{MNC}\right.$ as compared to $2 \times 10^{6} \mathrm{MNC}$ from patients), that were seeded for adherence, such that a morphologically similar cell population could be generated.

\section{Cell culture of IL-2-activated lymphocyte populations}

IL-2-activated T-cell lines were generated by culturing peripheral blood mononuclear cells in the presence of $10^{3} \mathrm{IU} / \mathrm{ml}$ of rhIL-2 (Proleukin/Aldesleukin NovartisBehring.de) in medium containing 10\% FCS in RPMI1640, $25 \mathrm{mM}$ Hepes using 24-well cluster plates. Cultures were split at cell densities of $>8 \times 10^{5} \mathrm{c} / \mathrm{ml}$.

Proliferative response of cultured APC and spontaneous caspase activity

Adherent cells were removed after 7 days, 14 days, 21 days and 28 days following culture initiation. Cells were seeded into 96-well round-bottomed microtiter plates and labeled with ${ }^{3} \mathrm{H}$-methyl thymidine $(0.5 \mu \mathrm{Ci} /$ well $)$ for $16 \mathrm{~h}$ and thymidine incorporation was determined by $B$-plate counting (Perkin Elmer.com). Spontaneous caspase activity and caspase activity induced by $3 \mathrm{~h}$ of staurosporin $(1 \mu \mathrm{M})$ activation were determined simultaneously.

\section{Electron microscopy}

For electron microscopy a high-pressure freezing method was applied as described by Buser and Walther (Buser and Walther 2008), with minor modifications. The cells were mounted onto sapphire discs and frozen via high pressure directly from the living state using a Compact- 01 
(Wohlwend GmbH, Sennwald, Switzerland). The freezesubstitution medium consisted of acetone with $0.2 \%$ osmium tetroxide, $0.1 \%$ uranyl acetate and 5\% water. Both chemically fixed and high-pressure frozen samples were embedded in EPON 812. Ultra thin sections (80 nm) were cut on a Leica microtome E, stained with $0.2 \%$ lead citrate, and imaged using an EM 10 (Zeiss, Oberkochen, Germany) at an accelerating voltage of $80 \mathrm{kV}$.

\section{Multiplex PCR for EBV-encoded genes}

Seven sets of oligonucleotide primers for the EBV genes targeted by the multiplex PCR are listed in Table 1. Aminolevulinate delta-synthase 1 (ALAS1) served as an internal control for the multiplex PCR (see below) and was purchased from Thermo Scientific (thermoscientific.com).

The Namalwa cell line (donated by Michael Pawlita, DKFZ, Heidelberg, Germany) served as a positive control for detecting virus genes while the Jurkat cell line (ATCC) served as a negative control. The genomic DNA from whole blood, isolated peripheral blood mononuclear cells and from cultured sepsis-APC was isolated by Qiagen columns (Qiagen.com). For multiplex PCR (25 $\mu \mathrm{l}) 2 \times$ master mix (Multiplex PCR kit, Qiagen.com) with HotStarTaq DNA polymerase, $\mathrm{MgCl}_{2}$, dNTPs, $1 \times$ PCR buffer, 200 pmol specific primer pairs for EBV genes or internal control (see Table 1), and $1 \mu$ l of sample genomic DNA as a template were mixed. PCR was carried out as follows: $95^{\circ} \mathrm{C} 15 \mathrm{~min}, 45$ cycles of denaturation $94^{\circ} \mathrm{C} 30 \mathrm{~s}$, annealing $62^{\circ} \mathrm{C} 90 \mathrm{~s}$ and extension $72^{\circ} \mathrm{C} 90 \mathrm{~s}, 1$ cycle final extension at $72^{\circ} \mathrm{C}$ for $10 \mathrm{~min}$ on the Palm-Cycler 96-PCR machine (Corbett Robotics; Qiagen.com).

Detection and analysis of PCR products

PCR products were loaded onto $8 \%$ polyacrylamide gels on a vertical electrophoresis system and run at $250 \mathrm{~V}$ for $30 \mathrm{~min}$. Gels were stained with silver nitrate and fixed with formaldehyde.

Fluorescence labeling of extracellular membranes and organelles

PKH67 green (Sigma 128 K0791) and PKH26 red (Sigma 077 K1774) were used for membrane labeling. Cells were washed in Hank's balanced salt solution (Invitrogen.com) and a cell pellet of $100 \mu \mathrm{l}$ was mixed with $100 \mu \mathrm{l}$ of dye buffer containing $2 \mu \mathrm{M}$ of dye. Cells were incubated for $5 \mathrm{~min}$ at room temperature, thereafter complete medium containing endotoxin-free FCS in RPMI1640, $25 \mathrm{mM}$ Hepes was added for a single washing step. Phagocytosis assays were performed with red fluorescent bacteria (a kind gift of Joerg Wiedenmann (Natl. Oceanographic Centre, Southampton, UK) and lysosomes were stained with Pepstatin A or Lysotracker Yellow (Invitrogen.com).

Confocal scanning microscopy

Cell specimens were appropriately labeled with fluorescent dyes and cultured in 15-well-slide ${ }^{\mathrm{TM}}$ from Ibidi ${ }^{\mathrm{TM}}$ (Ibidi.

Table 1 Primers for EBV multiplex PCR

\begin{tabular}{|c|c|c|c|c|}
\hline Target gene & Sequence & Length & Location in genomic gene & References \\
\hline \multirow[t]{2}{*}{ BNRF1 } & Sense 5'-GGAACCTGGTCATCCTTGC-3' & \multirow[t]{2}{*}{$74 \mathrm{bp}$} & $4733-4753$ & \multirow[t]{2}{*}{ (Niesters et al. 2000) } \\
\hline & Anti-sense 5'-ACGTGCATGGACCGGTTAAT-3' & & $4695-4679$ & \\
\hline \multirow[t]{2}{*}{ EBNA2 } & Sense 5'-CCAGAGGTAAGTGGACTT-3' & \multirow[t]{2}{*}{$124 \mathrm{bp}$} & $14614-1463$ & \multirow[t]{2}{*}{ (Saito et al. 1989) } \\
\hline & Anti-sense 5'-GACCGGTGCCTTCTTAGG-3' & & $14735-14718$ & \\
\hline \multirow[t]{2}{*}{ EBNA1 } & Sense 5'-GTCATCATCATCCGGGTCTC-3' & \multirow[t]{2}{*}{$220 \mathrm{bp}$} & $96822-96841$ & \multirow[t]{2}{*}{ (Telenti et al. 1990) } \\
\hline & Anti-sense 5'-TTCGGGTTGGAACCTCCTTG-3' & & $97091-97072$ & \\
\hline \multirow[t]{2}{*}{ LMP1 } & Sense 5'-GACATGGTAATGCCTAGAAG-3' & \multirow[t]{2}{*}{$259 \mathrm{bp}$} & $168130-168149$ & \multirow[t]{2}{*}{ (Midgley et al. 2003) } \\
\hline & Anti-sense 5'-GCGACTCTGCTGGAAATGAT-3' & & $168389-16$ & \\
\hline \multirow[t]{2}{*}{ BMLF } & Sense 5'-CACCACCTTGTTTTGACGGG-3' & \multirow[t]{2}{*}{$304 \mathrm{bp}$} & $83037-83056$ & \multirow[t]{2}{*}{ (Pedneault and Katz 1993) } \\
\hline & Anti-sense 5'-GTCAACCAACAAGGACACAT-3' & & $83340-83321$ & \\
\hline \multirow[t]{2}{*}{ EBNA3C } & Sense 5'-CAATCGCACCTGCAAGCGCTA-3' & \multirow[t]{2}{*}{$518 \mathrm{bp}$} & $100362-100383$ & \multirow[t]{2}{*}{ (Midgley et al. 2003) } \\
\hline & Anti-sense 5'-GACACCCATGAAACGCACGAAATC-3' & & $100806-100785$ & \\
\hline \multirow[t]{2}{*}{ BZLF1 } & Sense 5'-ATTGCACCTTGCCGCCACCTTTG-3' & \multirow[t]{2}{*}{$608 \mathrm{bp}$} & $103194-103180$ & \multirow[t]{2}{*}{ (Luo et al. 2005) } \\
\hline & Anti-sense 5'-CGGCATTTTCTGGAAGCCACCCGA-3' & & $102486-102463$ & \\
\hline \multirow[t]{2}{*}{ ALAS1 } & Sense 5'-CTGGGGCAGGTGGTACTAGA-3' & \multirow[t]{2}{*}{$804 \mathrm{bp}$} & $1161-1180$ & $\begin{array}{l}\text { Designed by Primer } 3 \\
\text { input } 0.4 .0\end{array}$ \\
\hline & Anti-sense 5'-TCATTGTGGCGGAAGATGTA-3' & & $1417-1397$ & online software \\
\hline
\end{tabular}


com). A confocal laser scanning microscope (Zeiss Meta 501) equipped with a $37^{\circ} \mathrm{C}$ incubation chamber and a PlanApochromat $63 \times$ oil immersion objective was used to monitor cell-cell interactions.

\section{Caspase measurement}

The Caspase-Glo ${ }^{\circledR}$ 3/7 luminescent assay measures cysteine- and aspartic acid-specific caspase- 3 and -7 activities. The luminogenic substrate contains the tetrapeptide sequence DEVD and generates a luminescent signal proportional to the amount of caspase activity generated in the cell incubation assay. The luminescent signal peaks in approximately one hour and is maintained for several hours (G8090, Promega.com). Luminescence was measured in a GLOMAX luminometer (Promega.com).

Flow cytometry

Cultured APC were washed in PBS and stained with monoclonal antibodies against the surface antigens (all from $\mathrm{BD}$ Biosciences, if not indicated differently) CD2, CD11c, CD14, CD163 (Abcam.com), CD95, CD205, CD207, CD208, CD209 (ebiosciences.com), CD282 (tolllike-receptor 2, TLR2) (Invivogen.com) CD284, (TLR4) (Morphosys.com), Trail receptor DR4 (ebiosciences.com), HLA-DR, CCR7, and CD68.

A fraction of the cultured cells was fixed in Perm/Fix solution (BD Biosciences), washed in Perm/Wash buffer (BD
Biosciences), underwent Fc-receptor blocking (by adding $10 \%$ of normal rabbit IgG (Dako)) and labeled for cytoplasmic antigens (TLR3 (Imgenix.com), CD178 (Alexis.biochemicals.com), and LC3, clone ab6717 (Abcam.com)). Antigen expression was quantified using flow cytometry (FACScalibur, BD Biosciences) and Cellquest software (BD Biosciences). Mean fluorescence intensities (MFI) were calculated by multiplying the percent positive cells $\mathrm{x}$ the mean channel of fluorescence detection.

\section{Results}

Properties of cultured antigen presenting cells isolated from peripheral blood of patients with severe sepsis and septic shock

The adherent fraction of peripheral blood mononuclear cells from patients with severe sepsis and septic shock gave rise to large, pleomorphic and floating antigen presenting cells. For convenience these phagocytic cells with dendritic or monocyte/macrophage differentiation antigens were called sepsis-derived antigen presenting cells, (sepsis-APC). The enriched APC cultured from adherent fractions of healthy blood donors were named blood donor-APC.

Remarkably, the phenotypes of individual patient's isolates were $>90-95 \%$ homogeneous in that all cells of a cell culture expressed the same antigens. Figure 1 summarizes the phenotype analysis of cultured blood donor-APC
Fig. 1 Expression pattern of cultured blood donor-APC and sepsis-APC. Cultured APC were harvested between day 14 and day 40 after culture initiation, and tested for surface marker expression analysis using flow cytometry. Cytoplasmic expression was determined for TLR3, CD178, CD207, and CD208 using fixed cells. MFI were calculated by multiplying percent positive cells $\mathrm{x}$ mean channel of fluorescence detection.

When less than 4 cultured APC isolates were available for testing, or when expression densities were very different, results are shown as scatter plots. An apparently separate subpopulation in the sepsis-APC group (TLR3 staining) has been encircled
Expression pattern of cultured blood donor-APC vs. sepsis-APC

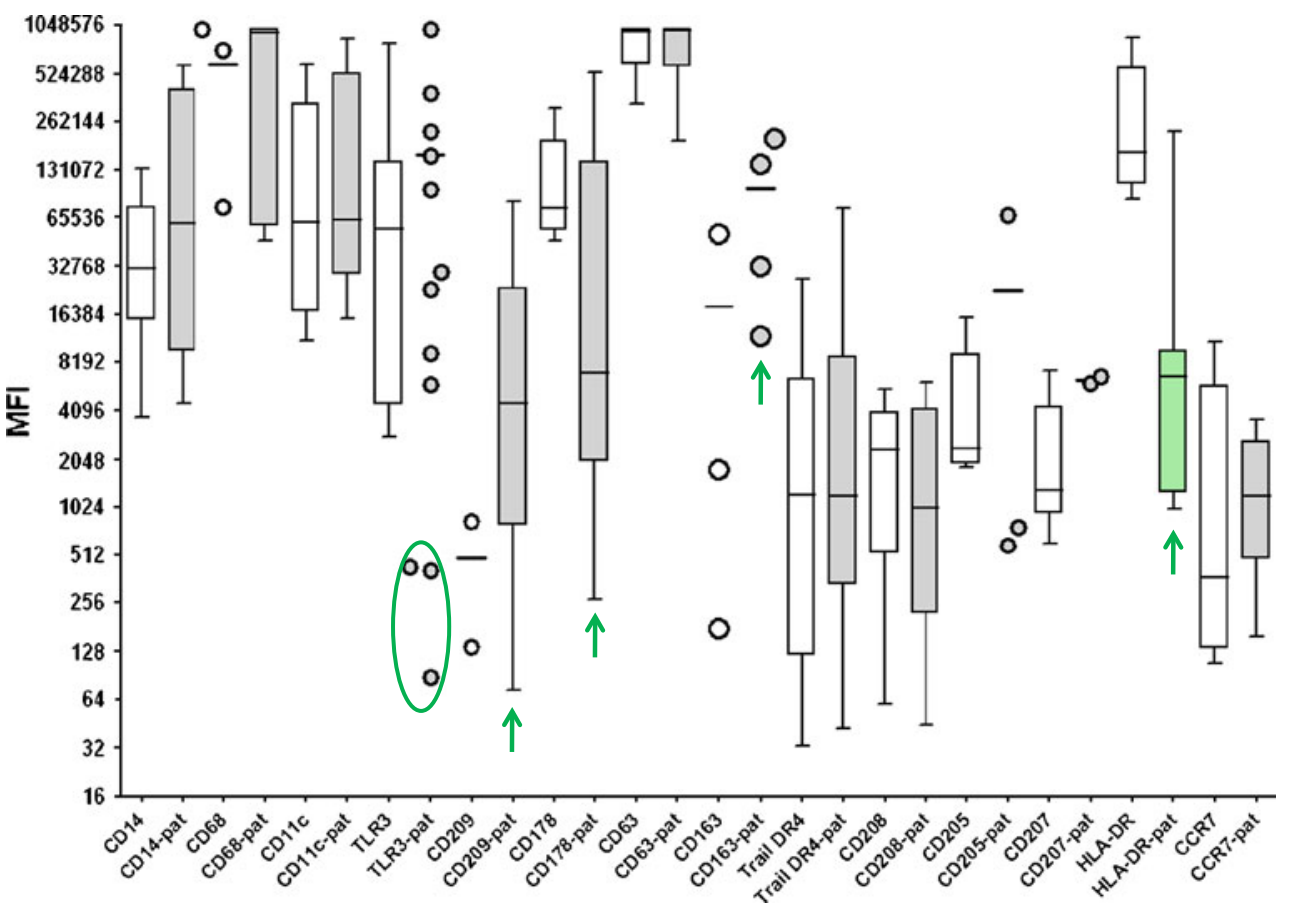


as compared to sepsis-APC. In each group, 3-10 different isolates were tested with some variation of the culture time (14 days to 40 days). Surface antigen expression was compatible with an immature, mostly CCR7-negative, myeloid dendritic cell type sharing some macrophage antigens, including CD68. There was no variation in antigen expression with respect to the culture time in vitro (data not shown). The dendritic cell phenotype was prominent due to low CD14, very high CD11c-expression and positivity for CD178 (Fas-ligand), CD205, CD207 (Langerin), CD208 (LAMP3) and CD209 (DC-SIGN). Remarkably, cultured blood donor-APC were phenotypically similar to cultured sepsis-APC. The most prominent difference was the low HLA-DR-expression in sepsis-APC. Furthermore, CD178 was less, but CD209 and CD163 were more prominent in sepsis-APC than in controls. A distinct subpopulation of sepsis-APC was indicated by its low TLR3 expression profile.

All isolates had a high capacity to ingest particles as well as bacteria. However, the ingested pathogens were not rapidly degraded, rather remained structurally intact in the cytoplasm of the cells for at least $13 \mathrm{~h}$. The delayed digestion of engulfed material is demonstrated in Fig. 2, where fluorescent bacteria were followed in pepstatinstained viable sepsis-APC. The persistence of nondegraded bacteria is possibly due to impaired phagolysosome fusion (Fig. 2a-c). In addition, many lysosomes in cultured APC were extremely large and only faintly stained with lysosome-specific dyes (Fig. 2d). The swollen lysosomes were often as large as the autophagy vacuoles. Autophagy vacuoles however appeared empty and never stained for either pepstatin or lysotracker dyes. Figure 3a,b shows the morphology of cultured sepsis-APC by conventional Wrights Giemsa staining, and similar to the fluorescence microscopy, the autophagy vacuoles appeared empty. When subjected to electron microscopy, the vacuoles were filled with engulfed cytoplasm which was less electron dense (Fig. 3c,d). In addition, some vacuoles also contained multivesicular bodies or mitochondria with various signs of structural degradation (Fig. 3c,d). Phenotypic results, such as low HLA-DR expression and immaturity imply that APC of patients with sepsis or septic shock are incapable of raising a $\mathrm{T}$ helper-1 response, even though sepsis is a unique, highly pro-inflammatory condition. To test this hypothesis, lymphocytes isolated from the same blood samples as the corresponding APC were propagated in medium containing high concentrations of IL-2. The proliferating lymphocytes were tested for surface antigens and cytokine production. The majority of the T lymphoblasts expressed CD4 ( $>65 \%$ of total lymphocytes) and secreted high amounts of IL-10 (Fig. 4).

In contrast, IL-2-cultured $\mathrm{T}$ lymphocytes from blood donors secreted only low amounts of IL-10 (Fig. 4) and a larger proportion expressed NK antigens (data not shown).
We therefore concluded that sepsis-APC appear to condition for an immunosuppressive microenvironment which supports the proliferation of IL-10-secreting T lymphocytes, whereas the morphologically and phenotypically similar blood donor-APC did not.

EBV detected in antigen presenting cells of patients with severe sepsis

Due to high IL-10 concentrations measured in $\mathrm{T}$ cell cultures with sepsis-APC we considered whether chronic EBV-infections may play a role in sepsis associated immune anergy. DNA prepared from cultured sepsis-APC and cultured blood donor-APC was subjected to a multiplex PCR designed to detect EBV encoded genes (Fig. 5). We found LMP1 in two and EBNA2 in seven out of nine cultured sepsis-APC isolates. For the other 5 marker genes none was found. In the corresponding whole blood specimen, prepared from the same patients, we detected LMP1 in none and EBNA2 in four DNA preparations. These results suggest that the EBV genes are found more often in enriched sepsis-APC. We also tested 56 whole blood samples of eight patients with septic shock and found EBNA2 in 31 individual blood samples.

In addition to the identification of EBV-specific genes, morphological evidence for persisting herpes viruses was provided by electron microscopical examination of sepsisAPC. Figure 6 shows virus particles in the extracellular space as well as in the cytoplasm of a sepsis-APC (Fig. 6).

P2RX7 stimulation by ATP causes inhibition of apoptosis

ATP is one of the danger-associated molecular patterns (DAMP) expected to play a major role in trauma-associated inflammation and cytokine storm. Dendritic cells express an important receptor for ATP, termed P2RX7, which constitutes the major calcium influx channel in these cells. We tested the effect of ATP on control blood donor APC as well as sepsis-APC. ATP at $1 \mathrm{mM}$ activated the P2RX7 and a characteristic electrophysiological response was detected by patch clamping (supplementary Fig. 1). The electrophysiological signal obtained was more than 10 times larger as compared to the P2RX7 signal of fresh dendritic cells or of dendritic cells differentiated from peripheral blood monocytes (data not shown). Moreover, P2RX7 activation by $1 \mathrm{mM}$ ATP stimulated ${ }^{3} \mathrm{H}$-thymidine incorporation (supplementary Fig. 2). The incorporation of radioactive nucleotide is, however, much extremely low since downcultured APC did not proliferate much. Exogeneous ATP also down-modulated the spontaneous caspase $3 / 7$ activity of cultured APC (Fig. 7). The low background caspase activity reflects the low proliferative capacity (suppl. Fig. 2) in that cultured APC exhibited neither significant prolifer- 
Fig. 2 Twenty-one-day cultured sepsis-APC derived from 3 different patients $(\mathbf{a}, \mathbf{b}, \mathbf{c})$ were incubated with red fluorescently-labeled E. coli for $3 \mathrm{~h}$, followed by labeling of lysosomes with pepstatin A (green fluorescent). None of the bacteria had entered the lysosomes. Individual phagocytes show swollen lysosomes (d), here stained with lysotracker yellow. Autophagy vacuoles (arrows) appear empty
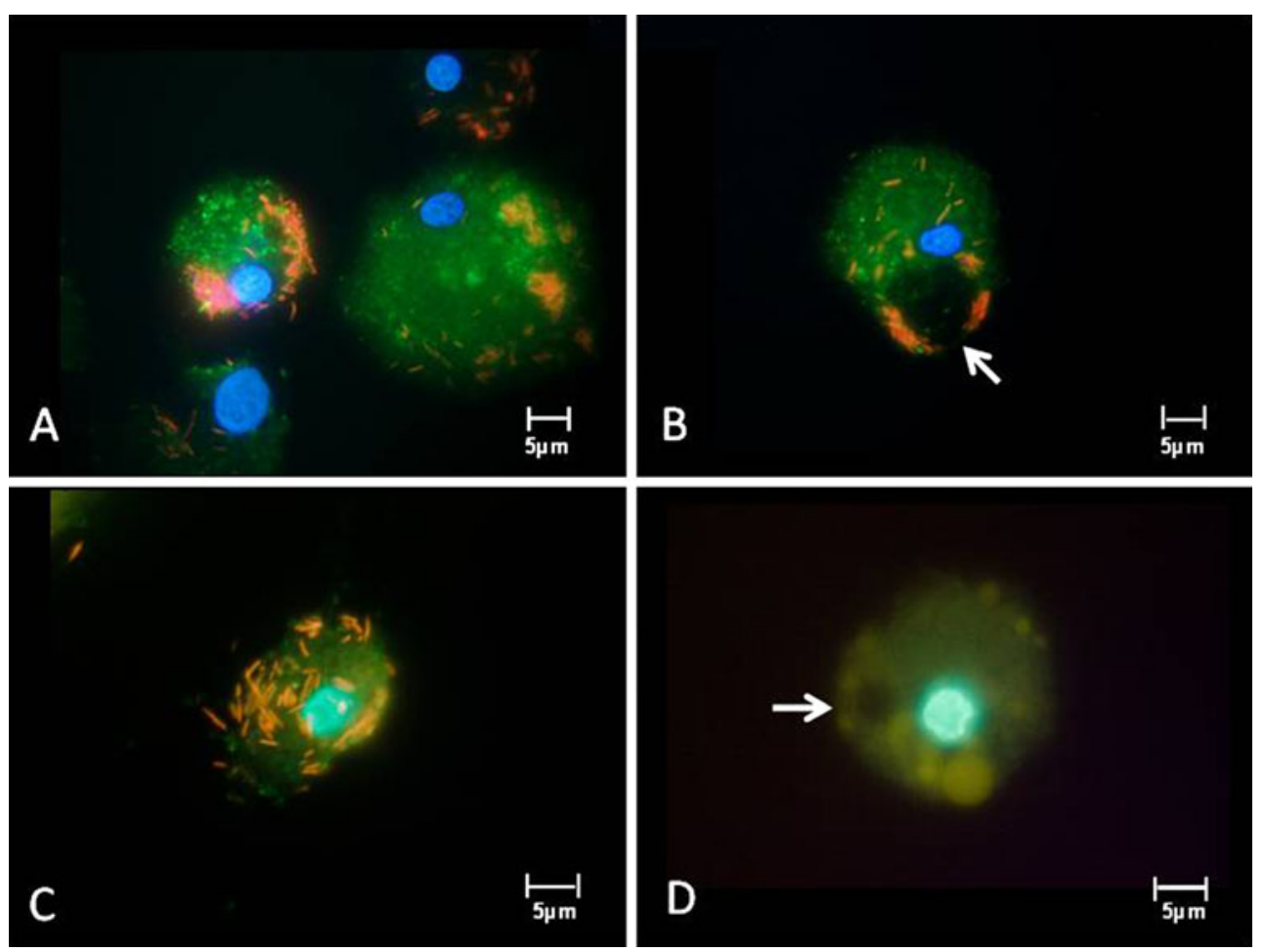

ation nor cell death during 14-40 days of in vitro culture. When cultured APC were labeled with a calcium-sensitive dye such as Fluo4-AM, stimulation with ATP caused a cytoplasmic and nuclear calcium response followed by the generation of autophagic vacuoles (supplementary Fig. 3). The same type of P2RX7 activation by ATP also increased the expression of LC-3 as determined by flow cytometry (supplementary Fig. 4). The functional organization of the P2RX7 ion channel with the inflammasome, the protein machinery which generates IL-1ß from pro-IL-1 $\beta$ in APC, was tested by priming sepsis-APC with endotoxin $(10 \mathrm{nM}$ LPS, overnight) and continued the culture with and without $1 \mathrm{mM}$ and $5 \mathrm{mM}$ ATP. Inflammasome activation in sepsisAPC was proven by the very large amounts of IL-1ß found in the supernatant (supplementary Fig. 5).

In summary, extracellular ATP contributes to the survival of IL-10-inducing sepsis-APC, and an immunosuppressive microenvironment may persist which also facilitates the persistence of infectious viruses such as EBV. When considering the potential counter regulatory mechanisms, it has previously been proposed that natural killer (NK) cells would be important in controlling immature dendritic cells. In mice, NK cells specifically regulate the number of immature dendritic cells present in lymph nodes (Giroux et al. 2007). In hemophagocytic diseases, genetic and acquired NK deficiencies appear to be responsible for the extensive proliferation of immature dendritic cells and the persistence of acute virus infections (Schneider et al. 2003). In our IL-2-stimulated cultures performed with adherencedepleted NK cells, it was difficult to enrich autologous
NK cells; which could in part be due to very low precursor frequencies or an as yet unexplained pathology. We therefore used the NK-92 cell line to test sepsis-APC as targets. Results displayed in Fig. 8a confirmed that NK-92 cells do indeed activate caspase $3 / 7$ in sepsis-APC targets. In order to prove, that the caspase released in NK-92/ sepsis-APC co-cultures was derived from the sepsis-APC target, confocal scanning microscopy was applied to study effector/target interactions. As shown in Fig. 8b, red fluorescent (PKH26-labeled) sepsis-APC and green fluorescent (PKH67-labeled) NK-92 interacted with prominent microvillous membrane protrusions before the lytic event took place (arrows in Fig. 8b). Sepsis-APC lysis was associated with prominent blebbing of the plasma membrane after 60 min of co-incubation with NK-92 (Fig. 8c). Although sepsis-APC were primed for death, they ingested plasma membrane-derived microparticles of the green fluorescent NK-92 effectors. In overlay images, the NK-92-derived membrane derivatives occur as yellow particles in the cytoplasm of the dying sepsis-APC (Fig. 8c, overlay image).

\section{Discussion}

This study describes how a major SIRS, followed by severe sepsis and septic shock results in the activation and persistence of immunosuppressive APC with an immature phenotype (Fig. 1). The high precursor frequency of this cell type facilitated our functional and phenotypic analysis. The dendritic nature of the cultured APC was proven by the 
Fig. 3 Autophagy in 21-day cultured sepsis-APC. Vacuolar structures (Wrights Giemsa stain: a, b). Cytoplasm containing multivesicular bodies (mvb) and mitochondria $(\mathrm{m})$ in various states of structural disintegration (c, bar: $500 \mathrm{~nm}$ )); enlarged mitochondria (d, bar: $100 \mathrm{~nm})$


extensive surface and cytoplasmic staining for differentiation antigens expressed by these cells. Interestingly, the same cell type could be enriched from healthy blood donors, when leukocyte fractions of a whole blood unit were used for enrichment. This fact is evidence for i) a nonmalignant nature and ii) for significantly increased precursor frequencies in patients encountering SIRS and other trauma-related stimulation in vivo. There was one subtle difference between APC-cultures of individual patients and the cultures derived from healthy blood donors: Some patients-derived APC-cultures contained very small lymphocyte-like cells in the vicinity of individual loosely adherent dendritic cells. After a few days, these small cells grew larger and made up small clusters with the original larger APC in the center. These small clusters (made up of 5-10 cells) were non-adherent and subsequently disseminated to individual floating APC with the characteristic morphology of an immature APC. However, when we made this observation on day 7 after culture initiation, other leukocytes were still present, and flow cytometry was not expected to support a reasonable analysis of this phenomenon. Since cultured APC lack the TNF-RI protein on the surface (data not shown), this property may hint at a mechanism for increased survival as described earlier (Funk et al. 2000). In addition to the myeloid-specific DC antigen CD11c, the APC enriched by cultures beyond 14 days displayed two differentiation profiles: One was the high expression of endocytosis-related antigens, and the other was the expression of dermal and epithelial-specific Langerin (CD207). It can be concluded that the cytokinefree and LPS-free (to low) culture conditions applied here favored the survival of this cell type without measurable proliferation after 14 days of in vitro culture. Only ATP led to a marginal effect on thymidine incorporation (supplementary Figure 2). When we tried to identify this cell type in healthy blood donors, about 20-100 times more blood was necessary to enrich a similar antigen presenting cell type which survived a prolonged time of in vitro culture. Surface 




Fig. 4 IL-10 concentrations of IL-2-cultured peripheral blood cells derived from healthy blood donors (upper part: healthy control) and from patients with SIRS and sepsis (lower part: inflammation-derived lymphocyte cultures). Each bar is the amount of IL-10 [pg/ml] secreted in individual cultures of $5-10 \times 10^{6}$ lymphocytes/ml during 4 days of in vitro culture

and cytoplasmic antigens were compared with sepsis-APC by flow cytometry. In both sepsis-APC and blood donor-control APC the enriched dendritic cell type was immature. The individual isolates were homogenous but there were differences between individual patients. Few isolates were tested after different culture periods and the individual phenotype appeared to be stable. Low CD14 and a number of functioning endocytosis receptors were found in all isolates. The CD205 antigen is an endocytic receptor mediating efficient processing and presentation of antigens in vivo, which leads the way to the induction of $\mathrm{T}$ cell immunity or tolerance (Bonifaz et al. 2002). CD207, Langerin is a C-type lectin, localized in the Birbeck granules of Langerhans cells which play a role in nonclassical antigen-processing (Bedoui et al. 2009). CD205 plays a unique role in virus control (de Witte et al. 2007) and collaborates with the classical DC-specific antigen CD209 (de Witte et al. 2008). Finally, we found a remarkable density of CD208, the lysosome-associated membrane glycoprotein 3 (LAMP3), which is highly homologous to the mannose receptor CD68, another strongly expressed antigen. CD208 is not only expressed in dendritic cells but also in type II pneumocytes and is transiently found in the MHC class II processing machinery (Arruda et al. 2006). Although CD208 is an early marker of dendritic cell maturation, its expression is related to arrested DC maturation in the context of malignancies (Elliott et al. 2007). Major differences between sepsis-APC and blood donor-APC were the significantly lower HLA-DR expression as well as a trend to express more CD209 (DC-SIGN), more CD163 and somewhat less CD178 in the case of sepsis-APC.

These 3 properties imply effects related to virus infections, which have been exemplarily studied by our multiplex EBV PCR. Herpes viruses down regulate HLAantigens as a major immune evasion strategy. In terms of $\mathrm{EBV}$, productive virus production is difficult to address experimentally. A previously proposed experimental model (Ressing et al. 2005) may support future studies to clarify whether cultured sepsis-APC does indeed produce infec-
Fig. 5 Multiplex PCR (lane 2) to detect various EBV encoded genes verified by singleplex PCR: ALAS1: housekeeping gene (lane 3). EBV genes occur by splice variants of the EBV genome. In the singleplex PCR, EBV-gene specific bands can be identified and are easily verified in the multiplex PCR lane 2. PCR products conform the expected length given in the Table (Materials and Methods, EBV specific PCR)

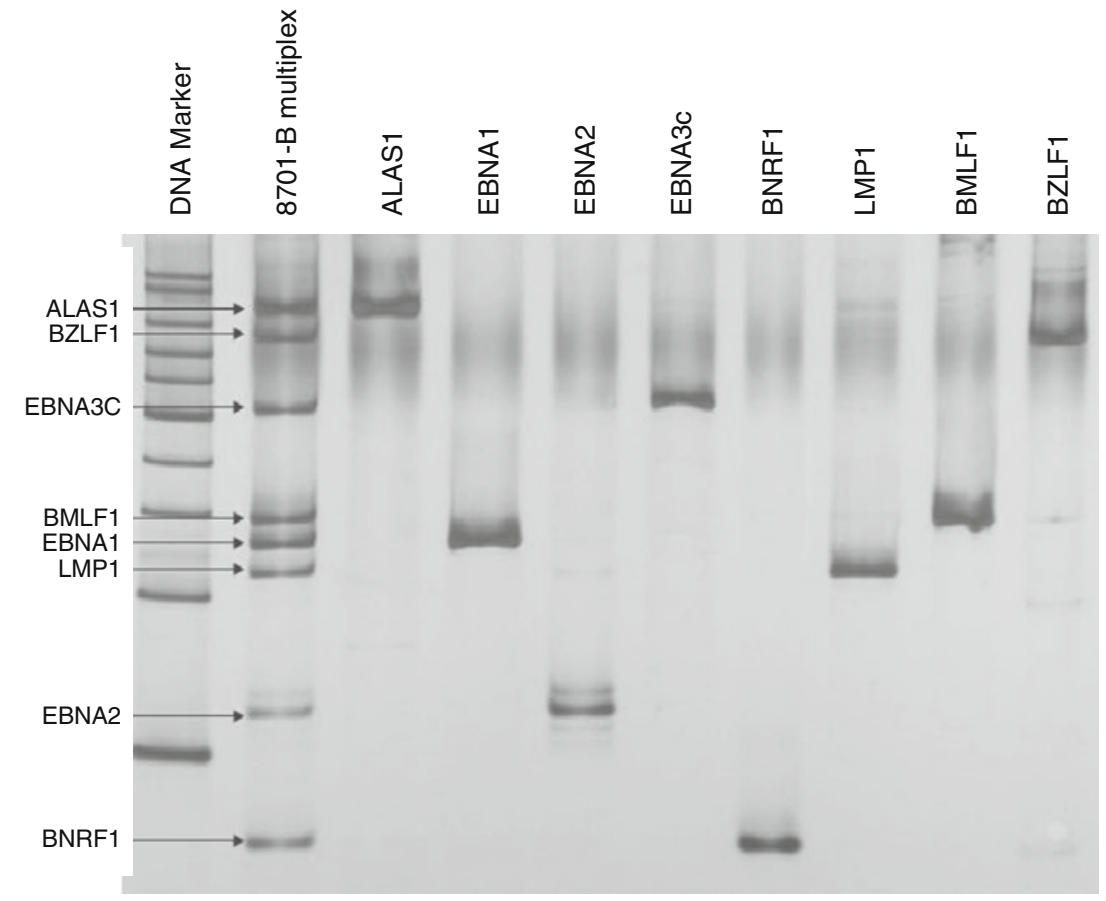


Fig. 6 Identification of herpes virus particles (right; arrows: inserted image) in 21-day cultured sepsis-APC (left: overview) of a patient with septic shock. The extracellular virus particle (arrow) shows spiny protrusions contacting the electron dense area of the plasma membrane. Intracellular virus particles (2 arrows) lack the outer capsid structures seen in the extracellular virus particle. The cytoplasm of the APC contains multiple autophagy vacuoles containing less electron dense cytoplasm

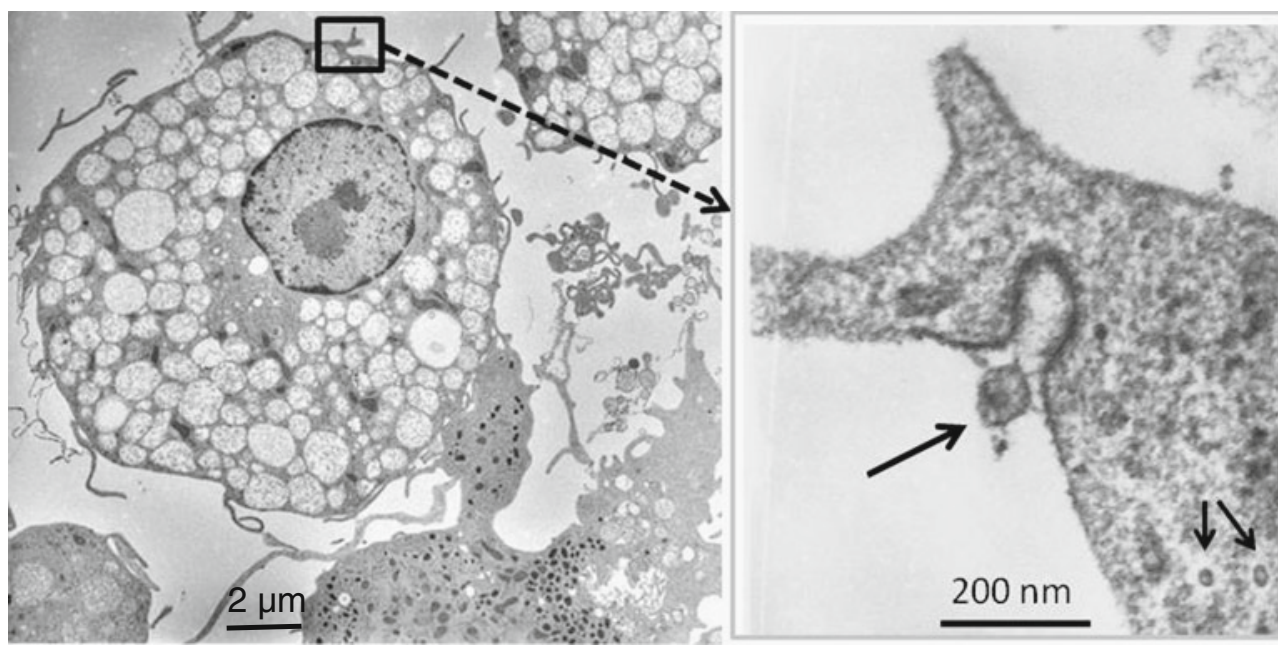

tious viruses as suggested in the present study (Fig. 5) here. The high level of DC-SIGN (CD209) expression is expected to affect a number of different virus infections other than HIV such as Herpes Virus 8 (HHV8) (Rappocciolo et al. 2006). Further DC-SIGN may cooperate with mannose-binding lectin structures such as MBL2. The relevance of MBL2 polymorphisms associated with susceptibility to virus infection (Acioli-Santos et al. 2008), including EBV (Friborg et al. 2010), are interesting in the context of the high expression density of CD68 by the cultured APC described here (Fig. 1).

In addition to phenotypic properties of sepsis-APC providing links to virus infections and endocytic activity,

\section{ATP downmodulates Caspase 3/7 in cultured APC}

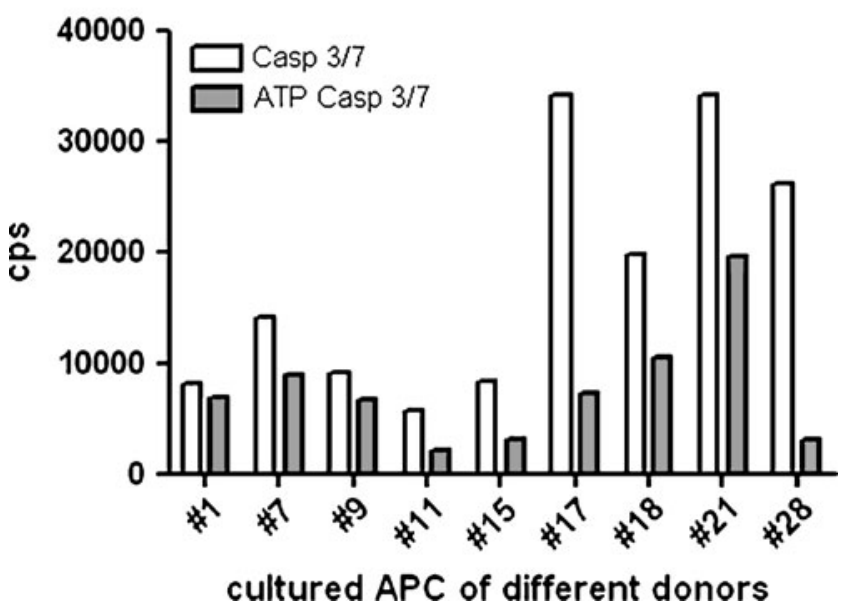

Fig. 7 Down-modulation of caspase $3 / 7$ after $3 \mathrm{~h}$ stimulation with $1 \mathrm{mM}$ ATP in cultured sepsis-APC of different patients. Different Sepsis-APC cultures (x-axis) were harvested and plated into 96-well non-transparent microtiter plates $\left(1 \times 10^{4} \mathrm{c} /\right.$ well $)$ in the presence or absence of $1 \mathrm{mM}$ ATP (pH 7.35 in RPMI 1640). After 3 h, lysates were measured for caspase activity (cps/well: y-axis) using chemiluminescence assays (Caspase Glo ${ }^{\circledR}$, Promega.com) morphological features have been addressed. In order to prove whether the large vacuoles of the cultured sepsisAPC play a role in phagocytosis, we used fluorescent bacteria and found that phagocytosed particles do not colocalize with these vacuoles. In addition, fusion of bacteria-loaded phagosomes with lysosomes was not observed within the first $3 \mathrm{~h}$ of co-incubation (Fig. 2). According to ultrastructural investigations, the vacuoles not only contained engulfed cytoplasm but also partiallydegraded mitochondria, suggesting that these organelles must have entered the phagolysosomal compartment (Fig. 4). This process of autophagy-related mitochondrial degradation has been called mitophagy (Kim et al. 2007) and plays a major role in the longevity of cells and tissues with autophagy. In inflammatory and infectious diseases, autophagy and impaired phagolysosome fusion processes have been described (Fortunato and Kroemer 2009), which culminate in, or are associated with immune dysfunction. We found many cultured sepsis-APC with enlarged and swollen lysosomes, an observation indicating increased $\mathrm{pH}$ in lysosomes, which is related to impaired antigen processing and T cell activation (Lem et al. 1999; Wubbolts et al. 1996). Since Lamp-2 has been recently described to play a major role in the acidification of the lysosome (Fortunato et al. 2009), and that molecular defects of this molecule have been detected in a number of diseases with impaired lysosomal functions (Ruivo et al. 2009), future studies in patients with severe sepsis and immune anergy should concentrate on the regulation of LAMP-2 expression as well as function.

Immature dendritic cells, possibly harboring viruses and expressing a low density of MHC antigens, are likely targets for NK-mediated cytolysis. However, high dose IL-2 cultures did not activate NK cells but rather induced a population of CD4 $\mathrm{T}$ cells secreting immune suppressive IL-10 (Fig. 4). IL-10 has been described as a cytokine which may be beneficial by attenuating the pro- 
Fig. 8 Cytolysis of cultured antigen presenting cells by NK-92 effector cells. NK-92 cells $\left(2 \times 10^{5}\right)$ were co-incubated with $2 \times 10^{4}$ cultured antigen presenting cells or $1 \times 10^{3} \mathrm{APC}$ (*) from different patients $(\mathrm{x}-$ axis). Caspase $3 / 7$ activity measured by chemiluminescense (cps) after $3 \mathrm{~h}$ of incubation (y-axis); open columns indicate the sum of spontaneous caspase activity of NK-92 plus cultured APC $\left(\sum\right)$, grey columns indicate caspase 3/7 activity of NK-92APC co-cultures (a).

Membrane-stained NK-92(green) and APC- (red) forming aggregates after $10 \mathrm{~min}$ of co-culture (b), and after $60 \mathrm{~min}$ intense blebbing occurs in sepsis-APC targets as well as phagocytosis of NK-92 membrane components (yellow vesicles in the sepsis-APC's cytoplasm (red/green overlay image in $\mathbf{c})$ ). Bar is $5 \mu \mathrm{m}$


inflammatory SIRS syndrome (Oberholzer et al. 2005), but may also be detrimental when specific immune activation is necessary to combat infectious antigens, as in severe sepsis (Hughes et al. 2009; Marie et al. 1998). In addition to bacteria and fungi, which invade the septic patient, the importance of virus reactivation syndromes has been recently documented (Limaye et al. 2008). Along these lines, we found Epstein Barr virus-specific genes in blood samples of patients with severe sepsis and septic shock. Approximately half of the patients' blood samples investigated by a specifically developed multiplex PCR were found to be positive for EBV-specific genes. The detection of EBV-encoded EBNA2 and LMP1 in cultures of sepsisAPC is of interest with respect to the concept that multiple viruses may be enriched in such immature and immunosuppressive APC. A preliminary study also demonstrated that EBV can even be detected in single cells using a low volume PCR protocol (uni-ulm.de/expane/quagsic). Although the nature of the herpes virus particles could not be proven on an ultrastructural level, it is highly likely that EBV particles were detected in sepsis-APC (Fig. 6).

In summary, massive tissue trauma appears to condition for the improved survival and/or accumulation of immature APC by DAMP receptor ligands such as ATP. DAMP signaling appears to be distinct from pathogen-derived signaling (PAMP), which plays a role later in the disease process of a critically ill patient. Unique DAMP receptors activate the inflammasome and caspase 1 (Dinarello 2009), the key enzyme responsible for the activation and release of IL-1ß (Martinon 2008; Martinon and Tschopp 2007). Data shown here support the idea that trauma-associated extracellular ATP serves as a danger ligand in cultured APC to activate a major calcium influx via P2XR7 (suppl. Fig. 3) (Franchi et al. 2007; Martinon 2008). Further proof has been provided by patch clamping (suppl. Fig. 1), by some activation of thymidine incorporation (suppl. Fig. 2), by a calcium flux-associated vacuole formation and microparticle release (suppl. Fig. 3), by increased LC3 expression (suppl.Fig. 4) and by ATP-stimulated IL-1ß production (suppl. Fig. 5). Previously, we described the activation of caspase 3 by ATP stimulation in whole blood (Schneider et al. 2006). In contrast to apoptosis induced by ATP in whole blood, we observed anti-apoptosis by ATP in a dendritic cell type which is rare in healthy blood donors. The here characterized trauma- and DAMP signaling-associated imbalance of immature APC may be responsible for SIRS-conditioned sepsis. Sepsis and acquired immune dysfunction would then be executed by IL-10-producing $\mathrm{T}$ cells.

The immunological control of immature antigen presenting cells by NK effectors has been described to be impaired in various diseases with an inflammatory phenotype (Fauriat et al. 2005; Walzer et al. 2005). When we investigated whether NK cells were able to recognize 
sepsis-APC, neither high dose IL-2 nor IL-15 (data not shown) generated autologous NK cell populations from patients' blood. To overcome this obstacle, we used NK-92 cells to test sepsis-APC as NK targets. Conveniently, NK-92 cells lack the majority of inhibitory receptors and could be used for all sepsis and control-APC. The majority of target cells were successfully lysed by NK-92 as shown by caspase 3 activation. However, microscopical examination suggests that cultured APC target cells endocytosed NK effectors before undergoing cytolysis. Due to this unique observation it may be hypothesized that low NK cell numbers or NK functional deficiency may in part explain the persistence of immunosuppressive APC and profound immune dysfunction in critically ill patients. Multiple surgical interventions in ICU patients may provide waves of extracellular ATP stimulating the DAMP signaling, and perpetuating the vicious cycle of P2RX7-mediated inflammation and autophagy in immunologically-uncontrolled APC.

Acknowledgements The authors thank the Landesstiftung Baden Wuerttemberg for support for the P2RX7 experiments and the International Histiocytosis Association for funding the work on the enrichment of cultured APC. Paul Walther is acknowledged for his support in electron microscopy, and Xuefang Ren for her support in establishing the multiplex PCR for EBV. We also thank Julian M Schneider for fluorescence microscopy and image analysis.

Competing interests statement None of the authors have any competing interests. EMS performed the cell culture experiments and wrote the ms; FL performed phenotype studies and data analysis of cultured APC; MRL performed the ultrastructural analysis; SF and PS contributed with caspase measurements and confocal microscopy; MEN performed multiplex PCR; KJF characterized P2RX7 electrophysiology; MHL and MEW contributed clinical evaluation and patients' diagnosis.

Open Access This article is distributed under the terms of the Creative Commons Attribution Noncommercial License which permits any noncommercial use, distribution, and reproduction in any medium, provided the original author(s) and source are credited.

\section{References}

Acioli-Santos B, Segat L, Dhalia R, Brito CA, Braga-Neto UM, Marques ET, Crovella S (2008) MBL2 gene polymorphisms protect against development of thrombocytopenia associated with severe dengue phenotype. Hum Immunol 69:122-128

Arruda LB, Sim D, Chikhlikar PR, Maciel M Jr, Akasaki K, August JT, Marques ET (2006) Dendritic cell-lysosomal-associated membrane protein (LAMP) and LAMP-1-HIV-1 gag chimeras have distinct cellular trafficking pathways and prime T and B cell responses to a diverse repertoire of epitopes. J Immunol 177:2265-2275

Bedoui S, Whitney PG, Waithman J, Eidsmo L, Wakim L, Caminschi I, Allan RS, Wojtasiak M, Shortman K, Carbone FR, Brooks AG, Heath WR (2009) Cross-presentation of viral and self antigens by skin-derived CD103+ dendritic cells. Nat Immunol 10:488-495

Bonifaz L, Bonnyay D, Mahnke K, Rivera M, Nussenzweig MC, Steinman RM (2002) Efficient targeting of protein antigen to the dendritic cell receptor DEC-205 in the steady state leads to antigen presentation on major histocompatibility complex class I products and peripheral CD8+ T cell tolerance. J Exp Med 196:1627-1638

Buser C, Walther P (2008) Freeze-substitution: the addition of water to polar solvents enhances the retention of structure and acts at temperatures around $-60^{\circ} \mathrm{C}$. J Microsc 230:268-277

de Witte L, Nabatov A, Pion M, Fluitsma D, de Jong MA, de Gruijl T, Piguet V, van Kooyk Y, Geijtenbeek TB (2007) Langerin is a natural barrier to HIV-1 transmission by Langerhans cells. Nat Med 13:367-371

de Witte L, Nabatov A, Geijtenbeek TB (2008) Distinct roles for DCSIGN+-dendritic cells and Langerhans cells in HIV-1 transmission. Trends Mol Med 14:12-19

Dinarello CA (2009) Immunological and inflammatory functions of the interleukin-1 family. Annu Rev Immunol 27:519-550

Efron P, Moldawer LL (2003) Sepsis and the dendritic cell. Shock 20:386-401

Efron PA, Martins A, Minnich D, Tinsley K, Ungaro R, Bahjat FR, Hotchkiss R, Clare-Salzler M, Moldawer LL (2004a) Characterization of the systemic loss of dendritic cells in murine lymph nodes during polymicrobial sepsis. J Immunol 173:3035-3043

Efron PA, Tinsley K, Minnich DJ, Monterroso V, Wagner J, Lainee P, Lorre K, Swanson PE, Hotchkiss R, Moldawer LL (2004b) Increased lymphoid tissue apoptosis in baboons with bacteremic shock. Shock 21:566-571

Elliott B, Scolyer RA, Suciu S, Lebecque S, Rimoldi D, Gugerli O, Musat E, Sharma RN, Lienard D, Keilholz U, Testori A, Eggermont A, MacKie R, Robert C, Cook M, Thompson JF, Angevin E, Spatz A (2007) Long-term protective effect of mature DC-LAMP+ dendritic cell accumulation in sentinel lymph nodes containing micrometastatic melanoma. Clin Cancer Res 13:3825-3830

Fauriat C, Moretta A, Olive D, Costello RT (2005) Defective killing of dendritic cells by autologous natural killer cells from acute myeloid leukemia patients. Blood 106:2186-2188

Fehrenbach E, Schneider ME (2006) Trauma-induced systemic inflammatory response versus exercise-induced immunomodulatory effects. Sports Med. 36:373-384

Fortunato F, Kroemer G (2009) Impaired autophagosome-lysosome fusion in the pathogenesis of pancreatitis. Autophagy 5:850-853

Fortunato F, Burgers H, Bergmann F, Rieger P, Buchler MW, Kroemer G, Werner J (2009) Impaired autolysosome formation correlates with Lamp-2 depletion: role of apoptosis, autophagy, and necrosis in pancreatitis. Gastroenterology 137:350-360, 360 e351-355

Franchi L, Kanneganti TD, Dubyak GR, Nunez G (2007) Differential requirement of $\mathrm{P} 2 \mathrm{X} 7$ receptor and intracellular $\mathrm{K}+$ for caspase-1 activation induced by intracellular and extracellular bacteria. $\mathrm{J}$ Biol Chem 282:18810-18818

Friborg JT, Jarrett RF, Koch A, Garred P, Freeland JM, Andersen A, Melbye M (2010) Mannose-binding lectin genotypes and susceptibility to epstein-barr virus infection in infancy. Clin Vaccine Immunol 17:1484-1487

Funk JO, Walczak H, Voigtlander C, Berchtold S, Baumeister T, Rauch P, Rossner S, Steinkasserer A, Schuler G, Lutz MB (2000) Cutting edge: resistance to apoptosis and continuous proliferation of dendritic cells deficient for TNF receptor-1. J Immunol 165:4792-4796

Giroux M, Yurchenko E, St-Pierre J, Piccirillo CA, Perreault C (2007) $\mathrm{T}$ regulatory cells control numbers of NK cells and CD8alpha+ immature dendritic cells in the lymph node paracortex. J Immunol 179:4492-4502

Hotchkiss RS, Karl IE (2003) The pathophysiology and treatment of sepsis. N Engl J Med 348:138-150

Hotchkiss RS, Coopersmith CM, McDunn JE, Ferguson TA (2009) The sepsis seesaw: tilting toward immunosuppression. Nat Med $15: 496-497$ 
Hughes SM, Amadi B, Mwiya M, Nkamba H, Tomkins A, Goldblatt D (2009) Dendritic cell anergy results from endotoxemia in severe malnutrition. J Immunol 183:2818-2826

Kim I, Rodriguez-Enriquez S, Lemasters JJ (2007) Selective degradation of mitochondria by mitophagy. Arch Biochem Biophys 462:245-253

Lem L, Riethof DA, Scidmore-Carlson M, Griffiths GM, Hackstadt T, Brodsky FM (1999) Enhanced interaction of HLA-DM with HLA-DR in enlarged vacuoles of hereditary and infectious lysosomal diseases. J Immunol 162:523-532

Limaye AP, Kirby KA, Rubenfeld GD, Leisenring WM, Bulger EM, Neff MJ, Gibran NS, Huang ML, Santo Hayes TK, Corey L, Boeckh M (2008) Cytomegalovirus reactivation in critically ill immunocompetent patients. Jama 300:413-422

Luo B, Wang Y, Wang XF, Liang H, Yan LP, Huang BH, Zhao P (2005) Expression of Epstein-Barr virus genes in EBV-associated gastric carcinomas. World J Gastroenterol 11:629-633

Marie C, Muret J, Fitting C, Losser MR, Payen D, Cavaillon JM (1998) Reduced ex vivo interleukin-8 production by neutrophils in septic and nonseptic systemic inflammatory response syndrome. Blood 91:3439-3446

Martinon F (2008) Detection of immune danger signals by NALP3. J Leukoc Biol 83:507-511

Martinon F, Tschopp J (2007) Inflammatory caspases and inflammasomes: master switches of inflammation. Cell Death Differ 14:10-22

Midgley RS, Bell AI, McGeoch DJ, Rickinson AB (2003) Latent gene sequencing reveals familial relationships among Chinese EpsteinBarr virus strains and evidence for positive selection of A11 epitope changes. J Virol 77:11517-11530

Niesters HG, van Esser J, Fries E, Wolthers KC, Cornelissen J, Osterhaus $\mathrm{AD}$ (2000) Development of a real-time quantitative assay for detection of Epstein-Barr virus. J Clin Microbiol 38:712-715

Oberholzer A, Oberholzer C, Efron PA, Scumpia PO, Uchida T, Bahjat K, Ungaro R, Tannahill CL, Murday M, Bahjat FR, Tsai V, Hutchins B, Moldawer LL, Laface D, Clare-Salzler MJ (2005) Functional modification of dendritic cells with recombinant adenovirus encoding interleukin 10 for the treatment of sepsis. Shock 23:507-515

Pedneault L, Katz BZ (1993) Comparison of polymerase chain reaction and standard Southern blotting for the detection of Epstein-Barr virus DNA in various biopsy specimens. J Med Virol 39:33-43
Rappocciolo G, Jenkins FJ, Hensler HR, Piazza P, Jais M, Borowski L, Watkins SC, Rinaldo CR Jr (2006) DC-SIGN is a receptor for human herpesvirus 8 on dendritic cells and macrophages. J Immunol 176:1741-1749

Ressing ME, Keating SE, van Leeuwen D, Koppers-Lalic D, Pappworth IY, Wiertz EJ, Rowe M (2005) Impaired transporter associated with antigen processing-dependent peptide transport during productive EBV infection. J Immunol 174:6829-6838

Ruivo R, Anne C, Sagné C, Gasnier B (2009) Molecular and cellular basis of lysosomal transmembrane protein dysfunction. Biochimica et Biophysica Acta (BBA) - Molecular Cell Research 1793:636-649

Saito I, Servenius B, Compton T, Fox RI (1989) Detection of EpsteinBarr virus DNA by polymerase chain reaction in blood and tissue biopsies from patients with Sjogren's syndrome. J Exp Med 169:2191-2198

Schneider EM, Lorenz I, Walther P, Janka-Schaub GE (2003) Natural killer deficiency: a minor or major factor in the manifestation of hemophagocytic lymphohistiocytosis? J Pediatr Hematol Oncol 25:680-683

Schneider EM, Vorlaender K, Ma X, Du W, Weiss M (2006) Role of ATP in trauma-associated cytokine release and apoptosis by P2X7 ion channel stimulation. Ann N Y Acad Sci 1090:245-252

Scumpia PO, McAuliffe PF, O'Malley KA, Ungaro R, Uchida T, Matsumoto T, Remick DG, Clare-Salzler MJ, Moldawer LL, Efron PA (2005) CD11c+ dendritic cells are required for survival in murine polymicrobial sepsis. J Immunol 175:3282-3286

Telenti A, Marshall WF, Smith TF (1990) Detection of Epstein-Barr virus by polymerase chain reaction. J Clin Microbiol 28:21872190

Walzer T, Dalod M, Robbins SH, Zitvogel L, Vivier E (2005) Naturalkiller cells and dendritic cells: "l'union fait la force". Blood 106:2252-2258

Willart MA, Lambrecht BN (2009) The danger within: endogenous danger signals, atopy and asthma. Clin Exp Allergy 39:12-19

Wubbolts R, Fernandez-Borja M, Oomen L, Verwoerd D, Janssen H, Calafat J, Tulp A, Dusseljee S, Neefjes J (1996) Direct vesicular transport of MHC class II molecules from lysosomal structures to the cell surface. J Cell Biol 135:611-622 